\title{
Performance Analysis of Emerging Data Analytics and HPC Workloads
}

\author{
Christopher S. Daley \\ Lawrence Berkeley National Laboratory \\ Berkeley, California \\ csdaley@lbl.gov \\ Prabhat \\ Lawrence Berkeley National Laboratory \\ Berkeley, California \\ prabhat@lbl.gov
}

\author{
Sudip Dosanjh \\ Lawrence Berkeley National Laboratory \\ Berkeley, California \\ sudip@lbl.gov \\ Nicholas J. Wright \\ Lawrence Berkeley National Laboratory \\ Berkeley, California \\ njwright@lbl.gov
}

\begin{abstract}
Supercomputers are increasingly being used to run a data analytics workload in addition to a traditional simulation science workload. This mixed workload must be rigorously characterized to ensure that appropriately balanced machines are deployed. In this paper we analyze a suite of applications representing the simulation science and data workload at the NERSC supercomputing center. We show how time is spent in application compute, library compute, communication and $\mathrm{I} / \mathrm{O}$, and present application performance on both the Intel Xeon and Intel Xeon-Phi partitions of the Cori supercomputer. We find commonality in the libraries used, I/O motifs and methods of parallelism, and obtain similar node-to-node performance for the base application configurations. We demonstrate that features of the Intel Xeon-Phi node architecture and a Burst Buffer can improve application performance, providing evidence that an exascale-era energy-efficient platform can support a mixed workload.
\end{abstract}

\section{KEYWORDS}

Workload characteristics, data analytics, big data, high performance computing

\section{ACM Reference Format:}

Christopher S. Daley, Sudip Dosanjh, Prabhat, and Nicholas J. Wright. 2017. Performance Analysis of Emerging Data Analytics and HPC Workloads. In Proceedings of PDSW-DISCS'17: Second foint International Workshop on Parallel Data Storage \& Data Intensive Scalable Computing Systems, Denver, CO, USA, November 12-17, 2017 (PDSW-DISCS'17), 6 pages.

https://doi.org/10.1145/3149393.3149400

\section{INTRODUCTION}

Supercomputers are increasingly being used to run a data analytics workload in addition to a traditional simulation science workload. This is happening because simulations and experiments are producing sufficiently large amounts of data that it is now impractical for many researchers to save and analyze data at their local institutions.

ACM acknowledges that this contribution was authored or co-authored by an employee contractor, or affiliate of the United States government. As such, the United States government retains a nonexclusive, royalty-free right to publish or reproduce this article, or to allow others to do so, for government purposes only.

PDSW-DISCS'17, November 12-17, 2017, Denver, CO, USA

(C) 2017 Association for Computing Machinery.

ACM ISBN 978-1-4503-5134-8/17/11 . \$ \$15.00

https://doi.org/10.1145/3149393.3149400
It is therefore critical for computing centers to continually assess the evolving workload to ensure that appropriately balanced machines are deployed. This can help answer an important question of whether a single architecture can support the needs of a broad simulation science and data analytics workload.

It is challenging to deploy a single architecture because the design space is large and there are many options in areas of compute, memory, storage and interconnect. In the compute space there are multi-core architectures consisting of "big" cores which provide higher clock speeds and features for tolerating latency in serial execution, many-core architectures consisting of many "small" cores which rely on highly parallel execution to deliver high performance, and hybrid CPU/GPU architectures which in-essence consist of a mixture of big and small cores. There are also new memory technologies such as High Bandwidth Memory (HBM) which provide greater aggregate memory bandwidth than standard DRAM DIMMs. The capacity of this technology is limited and so decisions must be made about whether it provides enough capacity or if it must be combined with another memory technology. The storage choices are also driven by performance and capacity trade-offs. This has motivated the inclusion of a Burst Buffer storage tier which is designed to provide a high performance buffer for I/O between compute nodes and a high capacity storage tier.

In this paper we analyze an application suite representative of the simulation science and data analytics workload run at the NERSC supercomputing center. The application suite covers simulation science (Quantum Espresso and Nyx), data analytics operating on simulation data sets (PCA and BD-CATS), and High Throughput Computing (HTC) data analytics operating on experimental data sets (SExtractor and PSFEx). We analyze performance on platforms with Intel Xeon-Phi (KNL) and Intel Xeon (Haswell) processors and show the extent that features of the Intel Xeon-Phi processor and a Burst Buffer can overcome the identified application performance bottlenecks. Our key findings are

- The BLAS, LAPACK and FFTW libraries are used extensively in many applications indicating that a single tuned software stack benefits both HPC and data analytics

- The base configurations of all applications run slower on the Intel Xeon-Phi node architecture than Intel Xeon. We demonstrate that running many HTC applications per compute node and strong scaling problems to fit within high 
bandwidth memory can improve performance on Intel XeonPhi, providing evidence that a single architecture can meet the needs of a mixed workload

- The Burst Buffer improves I/O time on a range of real application $\mathrm{I} / \mathrm{O}$ workloads by a factor of $2.3 \mathrm{x}$ to $26.7 \mathrm{x}$

\section{RELATED WORK}

There have been various workload studies evaluating application characteristics. Vetter et al. [20] evaluate a simulation science workload and find many common features including high fractions of integer instructions, low fractions of vector instructions and many collective communications with small message sizes typically less than 64 bytes. Wang et al. evaluate a commercial big data workload consisting of Spark and Hadoop implementations of various tasks such as word count, sort, page rank and grep. The authors find higher fractions of branch and integer instructions and less cache reuse compared to widely available benchmark suites. A qualitative evaluation of the DOE data analytics workload is provided in [16]

The Intel Xeon-Phi (KNL) is an energy-efficient node architecture on the path to exascale. Barnes et al. [7] present the performance of 20 applications selected as part of NERSC's early science program on KNL and Intel-Xeon (Haswell) processors. The authors describe optimization efforts and show improved performance on KNL for many applications. Byun et al. [11] compare two proxy data analytics applications and show that the KNL significantly improves the performance of the DGEMM kernel used by Matlab and Octave applications as well as the deep learning framework Caffe.

Burst Buffers are an additional tier in the storage hierarchy to accelerate I/O. Lui et al. [15] and Sato et al. [19] have shown how a Burst Buffer can improve the performance of a write workload and a checkpoint/restart workload, respectively. Bhimji et al. [10] compare the I/O performance of 5 applications and workflows when using the NERSC Burst Buffer. The authors show that the Burst Buffer can improve performance significantly but only after attention is paid to the number of concurrent I/O streams and I/O transaction size. Ovsyannikov et. al [17] demonstrate that the NERSC Burst Buffer can be used to efficiently couple a simulation science and data analytics application.

\section{METHODOLOGY}

In this section we describe the application suite and the architectural platforms used to evaluate performance.

\subsection{Applications}

Quantum Espresso [12] is a quantum chemistry application used for electronic structure calculations. It is written in Fortran and parallelized with MPI + OpenMP. We run the "large" test problem packaged with the APEX miniDFT application [1] (a Quantum Espresso mini-app) and use an optimized configuration of Quantum Espresso in which the number of band groups is set equal to the number of MPI ranks. Quantum Espresso uses the POSIX API to produce a checkpoint file per MPI rank and a charge density analysis file from the master rank only. We set disk_io='high' to write a checkpoint and analysis files every single computational step (i.e. each Self Consistent Field (SCF) cycle).
Nyx [6] is a Eulerian hydrodynamics and N-body gravity code used in computational cosmology. Nyx is written in $\mathrm{C}++$ and Fortran, parallelized with MPI + OpenMP, and uses the AMReX library to manage the block structured adaptive mesh. AMReX uses the POSIX API to produce a tunable number of output files ranging from 1 file per MPI rank to a single shared file [6, pp.20-21]. We run a Lyman-Alpha simulation on a uniform resolution mesh of $1024^{3}$ grid points for 5 time steps and produce a checkpoint and analysis data set every time step with 1 file per MPI rank.

PCA [2] (also referred to as mpi_pcavariants) performs Principle Component Analysis (PCA) on simulation data sets. The application is written in $\mathrm{C}$ and parallelized with MPI only. We run the application on a 2.16 TiB data set referred to as the Climate Forecast System Reanalysis Product data set [13, pp.4]. The data is stored in a single HDF5 file in one 2D double precision dataset and is read by the application using independent parallel I/O.

BD-CATS [18] is used to identify clusters of particles' in simulation data sets. It implements the Density-Based Spatial Clustering of Applications with Noise (DBSCAN) algorithm to assign particles to clusters. The application is written in $\mathrm{C}++$ and parallelized with MPI + OpenMP. We perform the clustering on a $32 \mathrm{GiB}$ cosmology data set consisting of $1024^{3}$ particles. The data is stored in a single HDF5 file in six 1D single-precision HDF5 datasets. At application completion the cluster ID is written to an 8-byte integer dataset in a new file. The data is read and written using collective parallel I/O.

SExtractor [9] provides information about the stars and galaxies found in astronomical images. The application is written in $\mathrm{C}$ and multithreaded with POSIX threads (although multithreading is currently disabled for correctness). It is run in two steps: first, image segmentation is used to identify the light sources in an image, and then second, light source targets are compared against different models of stars and galaxies to provide shape information for each target. We evaluate the longer-running second step on a Dark Energy Camera [4] data set consisting of an image file of size $32 \mathrm{MiB}$ and an an associated weight map file of size $16 \mathrm{MiB}$. Both files are in Flexible Image Transport System (FITS) file format.

PSFEx [8]: The Point Spread Function Extractor is used to estimate the spreading of light from stars and galaxies. It is written in $\mathrm{C}$ and parallelized with POSIX threads. We run it on the same data files as described for SExtractor.

\subsection{Architectural platforms}

All experiments are performed on Cori Phase 1 and Cori Phase 2 at NERSC: Cori Phase 1 consists of 2388 Intel Xeon nodes and Cori Phase 2 consists of 9668 Intel Xeon-Phi nodes. Each node of Cori Phase 1 contains two sixteen-core Intel Xeon E5-2698 v3 "Haswell" processors with two hyperthreads per core and a combined $128 \mathrm{GiB}$ of $2133 \mathrm{MHz}$ DDR4 memory. Each node of Cori Phase 2 contains one sixty-eight core Xeon-Phi 7250 "KNL" processor which can support 4 hardware threads per core. The processor has $16 \mathrm{GiB}$ of highbandwidth MCDRAM memory which can be directly addressed or used as a cache for the $96 \mathrm{GiB}$ of $2400 \mathrm{MHz}$ DDR4 main memory per node. Cori Phase 1 and Cori Phase 2 use the same Lustre scratch file system which consists of 248 Object Storage Targets (OSTs) and has a peak aggregate bandwidth of $744 \mathrm{GiB} / \mathrm{s}$ and a capacity of 30 PiB. Both partitions of Cori have access to a Cray DataWarp Burst 
Buffer [5] which is designed to accelerate application I/O [14]. The Burst Buffer consists of 288 Burst Buffer nodes and provides a peak aggregate bandwidth of $1.5 \mathrm{TiB} / \mathrm{s}$ and capacity of $1.8 \mathrm{PiB}$.

\section{RESULTS}

\subsection{Overall performance}

We ran the applications in their optimal MPI/OpenMP configurations on Cori Phase 2 to find out the amount of time spent in $\mathrm{I} / \mathrm{O}$, MPI communication, library compute and application compute. We use the node count recommended by the user/developer of each application and configure the nodes to use MCDRAM as a cache. The times are obtained from the Integrated Performance Monitoring (IPM) [3] profiler and manual measurements of library time. We follow the NERSC Lustre striping recommendations and use 24 OSTs for files of size 1-10 GiB and 72 OSTs for files over $100 \mathrm{GiB}$. The runtime configurations are shown in Table 1.

Figure 1 shows the breakdown of run time averaged over all MPI ranks. The results show that the capability data analytics applications, PCA and BD-CATS, spend over $40 \%$ of runtime in $\mathrm{I} / \mathrm{O}$. This is significantly higher than the HPC applications, Quantum Espresso and Nyx, despite them being configured to produce checkpoint and analysis files at the highest possible frequency. The amount of time spent in communication varies from zero in the HTC data analytics applications, SExtractor and PSFEx, to approximately $20 \%$ of time in Nyx and BD-CATS, to over $50 \%$ of time in Quantum Espresso. Quantum Espresso, Nyx and BD-CATS use a mixture of pointwise and collective communication calls where as PCA only uses collective communication calls. The dominant collective calls consist of all-to-all exchanges in Quantum Espresso, reductions over scalar variables and barriers in Nyx and BD-CATS, and relatively large reductions of $320 \mathrm{KiB} / \mathrm{rank}$ in PCA. The time spent in math libraries can be significant for some of the applications. In particular, PCA spends approximately $40 \%$ of time in the BLAS matrix-multiply function DGEMM and Quantum Espresso spends approximately $30 \%$ of time in the FFT functions DftiComputeForward and DftiComputeBackward. The PSFEx application also makes use of the same FFT functions but spends the majority of time in DGEMM and the LAPACK functions DSYTRS and DSYTRF.

Figure 2 shows the node-to-node performance gain of using Cori Phase 2 over Cori Phase 1. The relative performance is between $0.24 \mathrm{x}$ and $0.81 \mathrm{x}$ which indicates that all base application configurations perform worse on Intel Xeon-Phi. The performance loss is attributed to a combination of lack of thread and data parallelism.

\subsection{Opportunities for improving performance}

In this section we show results from optimization experiments on several of the studied applications. We use this to demonstrate that architectural features originally envisaged for HPC applications can also be used to improve the performance of data analytics applications. Our case studies consider a) launching many parallel tasks to utilize many small cores, b) strong scaling a problem to fit in MCDRAM, and c) accelerating I/O with a Burst Buffer.

4.2.1 Launching many parallel tasks to utilize many small cores. The high-throughput data analytics applications perform relatively poorly on KNL (SExtractor and PSFEx run $0.24 \mathrm{x}$ and $0.33 \mathrm{x}$ as fast

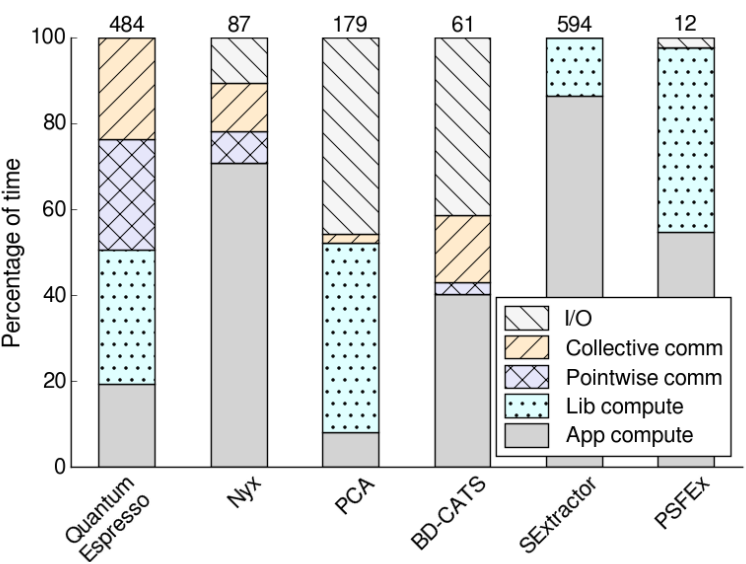

Figure 1: Breakdown of time on Cori Phase 2. The value above each bar is the absolute run time in seconds. Quantum Espresso and Nyx values are time per step; production simulations run for $\sim 50$ and 1000 steps, respectively.

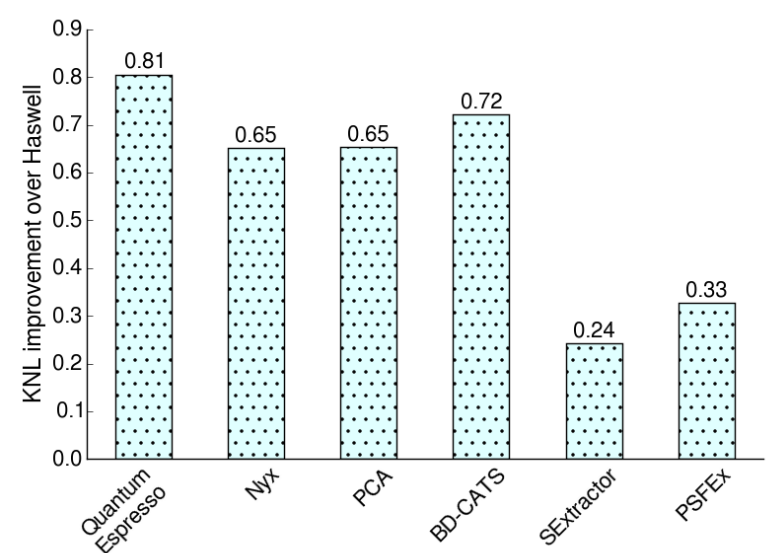

Figure 2: Relative application performance on Cori Phase 2 versus Cori Phase 1. Values less than 1.0 indicate that the applications run slower on the KNL nodes of Cori Phase 2. The time to solution excludes time in $\mathrm{I} / \mathrm{O}$.

as Haswell). This is because SExtractor is serial and PSFEx only has a thread-speedup of $16 \mathrm{x}$ on 64 cores. We experiment with adding a MPI wrapper around both applications to simulate the launch of independent tasks operating on different data sets concurrently. This step would normally be performed by workflow management software.

Figure 3 shows that running multiple SExtractor and PSFEx applications on a single compute node improves average time to solution on both architectures The most significant result is that the KNL to Haswell ratio is improved from $0.24 \mathrm{x}$ to $0.75 \mathrm{x}$ in SExtractor and from $0.33 \mathrm{x}$ to $1.02 \mathrm{x}$ in PSFEx when running at the optimal high throughput concurrency. The cost of using these configurations is that memory footprint increases from $0.6 \mathrm{GiB}$ to $84.5 \mathrm{GiB}$ for SExtractor and from $0.2 \mathrm{GiB}$ to $5.0 \mathrm{GiB}$ for PSFEx on KNL. This indicates that memory requirements can be significant when running independent serial applications on many small cores of KNL. 


\begin{tabular}{|c|c|c|c|c|c|c|}
\hline & Quantum Espresso & Nyx & PCA & BD-CATS & SExtractor & PSFEx \\
\hline Compute nodes & 96 & 16 & 50 & 16 & 1 & 1 \\
\hline I/O motif & FPP & FPP & SSF (ind.) & SSF (coll.) & FPP & FPP \\
\hline I/O API & POSIX & POSIX & HDF5 & HDF5 & POSIX & POSIX \\
\hline $\mathrm{I} / \mathrm{O}(\mathrm{GiB})$ & 7.2 & 1465.5 & 2211.2 & 20.0 & 0.1 & 0.1 \\
\hline Lustre stripe count & 1 & 1 & 72 & 24 & 1 & 1 \\
\hline Processes per node & 2 & 8 & 16 & 16 & 1 & 1 \\
\hline Threads per process on KNL & 32 & 32 & 4 & 16 & 1 & 128 \\
\hline Threads per process on Haswell & 16 & 8 & 4 & 4 & 1 & 64 \\
\hline Memory footprint per process (GiB) & 21.2 & 7.6 & 2.8 & 0.4 & 0.6 & 0.2 \\
\hline
\end{tabular}

Table 1: Application configurations. The I/O motifs include File Per Process (FPP) and Single Shared File (SSF) in independent and collective access modes. The memory footprint per process is obtained on KNL.

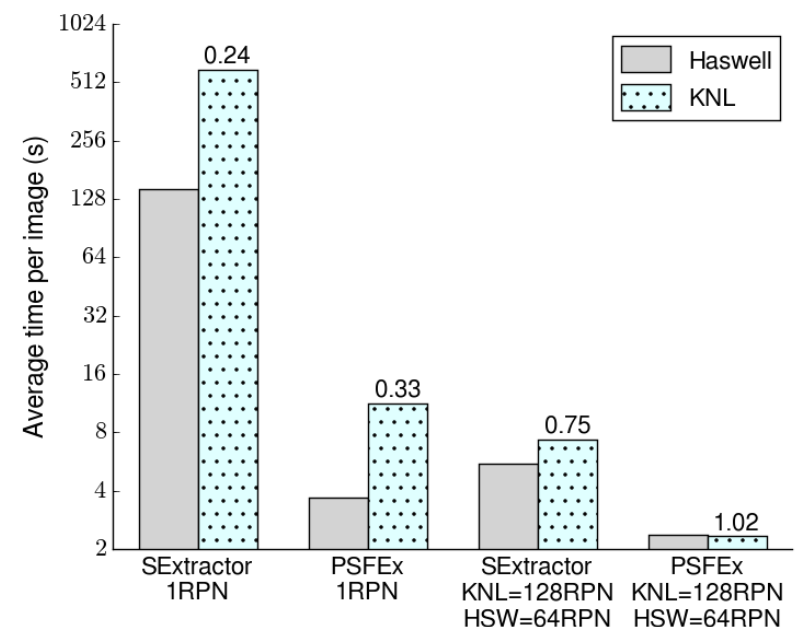

Figure 3: Average time to analyze a single input file when using 1 rank per node and multiple ranks per node. The values above the bars show the performance improvement when using Cori Phase 2 KNL nodes.

4.2.2 Strong scaling a problem to fit in MCDRAM. The base configuration of PCA uses 50 compute nodes and has a memory footprint of $44.7 \mathrm{GiB}$ per node. It is run with $16 \mathrm{MPI}$ ranks per node and spends $44 \%$ of total run time in the DGEMM function (see Figure 1). We are not able to improve performance by adding more ranks per node because of memory bandwidth saturation. This puzzling observation is because PCA uses the DGEMM function to multiply a matrix by a vector, i.e. using a DGEMM to perform a DGEMV. We experiment with adding compute nodes to reduce the memory footprint per node and fit within the $16 \mathrm{GiB}$ of MCDRAM on Cori Phase 2.

Figure 4 shows the strong scaling performance of PCA. The KNL speedup is super-linear at 100 and 200 nodes indicating that it is more efficient to use this concurrency than the 50-node base configuration. The KNL performance is almost the same as the Haswell performance at 200 and 400 nodes because of reduced time in the DGEMM function. The KNL performance suffers at 800 nodes because of increased significance of non-DGEMM code. The non-DGEMM code is serial and so performs poorly on 16 small cores of KNL compared to 16 big cores of Haswell.

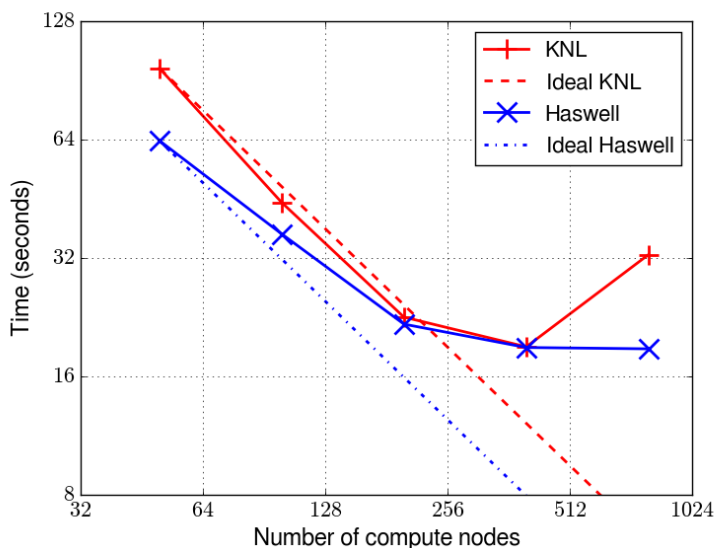

Figure 4: Strong scaling performance of PCA on Cori Phase 1 and Cori Phase 2 when using 16 MPI ranks per node. The memory footprint is $44.7,22.6,11.5,6.0$ and $3.3 \mathrm{GiB} /$ node at $50,100,200,400$ and 800 nodes, respectively. The time to solution excludes time in $\mathrm{I} / \mathrm{O}$.

4.2.3 Accelerating I/O with a Burst Buffer. The Nyx, PCA and BD-CATS applications spend $11 \%, 46 \%$ and $41 \%$ of run time in I/O. We experiment with performing the I/O against the Burst Buffer to determine whether the Burst Buffer can accelerate File Per Process (FPP) writes of Nyx, Single Shared File (SSF) independent reads of PCA and SSF collective reads of BD-CATS.

Table 2 shows the I/O performance of Nyx, PCA and BD-CATS on the Scratch file system and the Burst Buffer, where we use the fastest time from at least two trials per application. The Burst Buffer improves I/O performance over the Scratch file system by $2.3 \mathrm{x}$ to $26.7 \mathrm{x}$. The improvement of 26.7 is significant and was likely due to poor Scratch file system performance over multiple runs. We also show the percentage of achieved Burst Buffer peak bandwidth, which was calculated by multiplying the number of Burst Buffer nodes used in the allocation by the theoretical peak of $5.7 \mathrm{GiB} / \mathrm{s} /$ Burst Buffer node 1 . The results show that the three applications achieve between $15 \%$ and $34 \%$ of the theoretical Burst Buffer peak bandwidth, indicating effective usage of the Burst Buffer. We note that all configurations used fewer compute nodes than Burst Buffer nodes. It is necessary

${ }^{1}$ We are only able to achieve a peak write performance of $4.5 \mathrm{GiB} / \mathrm{s} /$ Burst Buffer node in I/O benchmark tests performed in October 2017. 
to use more compute nodes (and application I/O streams) to get closer to the peak performance of the Burst Buffer allocation.

\begin{tabular}{lcrr}
\hline & Nyx & PCA & BD-CATS \\
\hline Compute nodes & 16 & 50 & 16 \\
BB nodes & 32 & 72 & 24 \\
BB improvement & 2.3 & 3.4 & 26.7 \\
\% BB bandwidth peak & 34 & 23 & 15
\end{tabular}

Table 2: I/O performance when using the Lustre Scratch file system and the Burst Buffer (BB) on Cori Phase 2. The number of Burst Buffer nodes is equal to the Lustre stripe count for all configurations except for $\mathrm{Nyx}$ which used a Lustre stripe count of 1 .

\section{DISCUSSION}

There is some commonality in the compute needs of simulation science and data analytics applications. Four of the six applications use BLAS, LAPACK and FFTW libraries (through Intel MKL) and spend as much as $40 \%$ of run time in these math libraries. This indicates that the same tuned software stack can benefit simulation science and data analytics applications. The HTC data analytics have no process-level parallelism and perform poorly on Cori Phase 1 and Cori Phase 2. We demonstrate that performance can be improved by launching multiple independent tasks per compute node. This improves compute-node utilization on both architectures and achieves a node-to-node performance similar to the other applications. Overall there is a lack of thread scaling and data parallelism in all applications and performance is better on the big cores of Cori Phase 1 compared to Cori Phase 2.

The memory footprint per process varies from $0.2 \mathrm{GiB}$ in PSFEx to $21.2 \mathrm{GiB}$ in Quantum Espresso. Four applications, Quantum Espresso, Nyx, PCA and the MPI-wrapped SExtractor, have a memory footprint per compute node which exceeds MCDRAM memory capacity on Cori Phase 2 . The significance of this is application dependent: PCA performance improves dramatically when the memory footprint is less than MCDRAM capacity, where as SExtractor throughput improves with intra-node concurrency despite exceeding MCDRAM capacity. The PCA application benefits from fitting in MCDRAM because it is both memory bandwidth limited and makes poor use of two-level memory. In applications with this characteristic, this can present an optimization trade-off between increasing intra-node concurrency to make use of many small cores and staying within MCDRAM memory capacity. More work is needed to evaluate the performance benefit of a two-level memory in a production workload. The memory capacity needed to run SExtractor efficiently is approximately $1.0 \mathrm{GiB} /$ core. This means that sufficient memory capacity must be available to run the breadth of a data analytics workload.

The capability data analytics applications spend more than $40 \%$ of run time in $\mathrm{I} / \mathrm{O}$ which is significantly more than the simulation science and HTC data analytics applications. These applications read data from shared HDF5 files with extremely simple data layouts, where data for each MPI rank is obtained by a single subarray read from each HDF5 dataset. We demonstrate that the Burst Buffer can be used to improve the performance of these structured reads by $3.4 \mathrm{x}$ and $26.7 \mathrm{x}$ as well as improving the performance of Nyx structured writes by $2.3 \mathrm{x}$. This indicates that investments in fast storage layers can benefit both HPC and data analytics applications. The data analytics applications are read-dominated and are best supported by a Burst Buffer architecture and accompanying API which supports pre-staging the input data before the application starts. The remaining applications, Quantum Espresso, SExtractor and PSFEx, only spend a small amount of time in file-per-process accesses to small files.

\section{CONCLUSIONS}

In this paper we have analyzed a suite of applications representing the simulation science and data analytics workload at NERSC. We have presented a breakdown of how time is spent in the different applications and have shown relative performance on Cori Phase 1 and Cori Phase 2. We have found that the simulation science and data analytics applications use common math and I/O libraries, programming languages and methods of parallelism. We have also found that the base application configurations perform worse on the Intel Xeon-Phi architecture, however, we have shown how features of the Intel Xeon-Phi architecture and a Burst Buffer can improve application performance. Our analysis and optimization experiments remove some uncertainty about the characteristics of data analytics applications and gives us reason to believe that an energy-efficient architecture in the exascale time frame can support a mixed workload. We will continue to monitor the evolving NERSC workload, e.g. increased use of machine learning techniques, to understand whether it will become beneficial to deploy different specialized architectures.

\section{ACKNOWLEDGMENTS}

The authors would like to thank Jack Deslippe, Zarija Lukić, Alex Gittens, Mostofa Ali Patwary and Peter Nugent for providing the application test problems used in this paper.

This work was supported by Laboratory Directed Research and Development (LDRD) funding from Berkeley Lab, provided by the Director, Office of Science, Office of Advanced Scientific Computing Research (ASCR) of the U.S. Department of Energy under Contract No. DE-AC02-05CH11231. This research used resources of the National Energy Research Scientific Computing Center, a DOE Office of Science User Facility supported by the Office of Science of the U.S. Department of Energy under Contract No. DE-AC02-05CH11231.

\section{REFERENCES}

[1] 2016. MiniDFT. http://www.nersc.gov/research-and-development/apex/ apex-benchmarks/minidft/; accessed 7 September 2017. (March 2016).

[2] 2016. MPI implementations of PCA and RPCA. https://github.com/alexgittens/ mpi_pcavariants; accessed 7 September 2017. (2016)

[3] 2017. IPM. https://github.com/nerscadmin/IPM; accessed 7 September 2017. (2017).

[4] 2017. The DECam Legacy Survey. http://legacysurvey.org/; accessed 7 September 2017. (2017).

[5] 2017. XC Series DataWarp User Guide. Technical Report CLE 6.0.UP04 S-2558. Cray. http://docs.cray.com/PDF/XC_Series_DataWarp_User_Guide_CLE60UP04_ S-2558.pdf; accessed 7 September 2017.

[6] Ann S. Almgren, John B. Bell, Mike J. Lijewski, Zarija Lukić, and Ethan Van Andel. 2013. Nyx: A Massively Parallel AMR Code for Computational Cosmology. The Astrophysical fournal 765, 1 (2013), 39. http://stacks.iop.org/0004-637X/765/i=1/ $\mathrm{a}=39$

[7] T. Barnes, B. Cook, J. Deslippe, D. Doerfler, B. Friesen, Y. He, T. Kurth, T. Koskela, M. Lobet, T. Malas, L. Oliker, A. Ovsyannikov, A. Sarje, J. L. Vay, H. Vincenti, S. 
Williams, P. Carrier, N. Wichmann, M. Wagner, P. Kent, C. Kerr, and J. Dennis. 2016. Evaluating and Optimizing the NERSC Workload on Knights Landing. In 2016 7th International Workshop on Performance Modeling, Benchmarking and Simulation of High Performance Computer Systems (PMBS). 43-53. https://doi.org/ 10.1109/PMBS.2016.010

[8] E. Bertin. 2011. Automated Morphometry with SExtractor and PSFEx. In Astronomical Data Analysis Software and Systems XX (ADASSXX) (Astronomical Society of the Pacific Conference Series), I.N. Evans, A. Accomazzi, D.J. Mink, and A.H. Rots (Eds.), Vol. 442. 435.

[9] E. Bertin and S. Arnouts. 1996. SExtractor: Software for source extraction. Astronomy \& Astrophysics, Supplement 117 (June 1996), 393-404.

[10] W. Bhimji et al. 2016. Accelerating Science with the NERSC Burst Buffer Early User Program. In Cray User Group CUG. https://cug.org/proceedings/cug2016 proceedings/includes/files/pap162.pdf

[11] C. Byun, J. Kepner, W. Arcand, D. Bestor, B. Bergeron, V. Gadepally, M. Houle, M. Hubbell, M. Jones, A. Klein, P. Michaleas, L. Milechin, J. Mullen, A. Prout, A. Rosa, S. Samsi, C. Yee, and A. Reuther. 2017. Benchmarking Data Analysis and Machine Learning Applications on the Intel KNL Many-Core Processor. ArXiv e-prints (July 2017). arXiv:cs.PF/1707.03515

[12] Paolo Giannozzi, Stefano Baroni, Nicola Bonini, Matteo Calandra, Roberto Car Carlo Cavazzoni, Davide Ceresoli, Guido L Chiarotti, Matteo Cococcioni, Ismaila Dabo, Andrea Dal Corso, Stefano de Gironcoli, Stefano Fabris, Guido Fratesi, Ralph Gebauer, Uwe Gerstmann, Christos Gougoussis, Anton Kokalj, Michele Lazzeri, Layla Martin-Samos, Nicola Marzari, Francesco Mauri, Riccardo Maz zarello, Stefano Paolini, Alfredo Pasquarello, Lorenzo Paulatto, Carlo Sbraccia, Sandro Scandolo, Gabriele Sclauzero, Ari P Seitsonen, Alexander Smogunov, Paolo Umari, and Renata M Wentzcovitch. 2009. QUANTUM ESPRESSO: a modular and open-source software project for quantum simulations of materials. Fournal of Physics: Condensed Matter 21, 39 (2009), 395502 (19pp) http://www.quantum-espresso.org

[13] Alex Gittens, Aditya Devarakonda, Evan Racah, Michael F. Ringenburg, Lisa Gerhardt, Jey Kottalam, Jialin Liu, Kristyn J. Maschhoff, Shane Canon, Jatin Chhugani, Pramod Sharma, Jiyan Yang, James Demmel, Jim Harrell, Venkat Krishnamurthy, Michael W. Mahoney, and Prabhat. 2016. Matrix Factorization at Scale: a Comparison of Scientific Data Analytics in Spark and C+MPI Using Three Case Studies. CoRR abs/1607.01335 (2016). http://arxiv.org/abs/1607.01335

[14] D. Henseler, B. Landsteiner, D. Petesch, C. Wright, and N.J. Wright. 2016. Architecture and Design of Cray DataWarp. In Cray User Group CUG. https: //cug.org/proceedings/cug2016_proceedings/includes/files/pap105.pdf

[15] N. Liu, J. Cope, P. Carns, C. Carothers, R. Ross, G. Grider, A. Crume, and C. Maltzahn. 2012. On the role of burst buffers in leadership-class storage systems. In IEEE 28th Symposium on Mass Storage Systems and Technologies (MSST). 1-11. https://doi.org/10.1109/MSST.2012.6232369

[16] Office of Advanced Scientific Computing Research. DOE Office of Science. 2015. Management, Visualization, and Analysis of Experimental and Observational Data (EOD). The Convergence of Data and Computing Workshop Final Report Technical Report LBNL-1005155. https://science.energy.gov/ /media/ascr/pdf/ programdocuments/docs/ascr-eod-workshop-2015-report_160524.pdf; accessed 7 September 2017.

[17] A. Ovsyannikov, M. Romanus, B. V. Straalen, G. H. Weber, and D. Trebotich. 2016 Scientific Workflows at DataWarp-Speed: Accelerated Data-Intensive Science Using NERSC's Burst Buffer. In 2016 1st foint International Workshop on Parallel Data Storage and data Intensive Scalable Computing Systems (PDSW-DISCS). 1-6. https://doi.org/10.1109/PDSW-DISCS.2016.005

[18] Md. Mostofa Ali Patwary, Pradeep Dubey, Suren Byna, Nadathur Rajagopalan Satish, Narayanan Sundaram, Zarija Lukić, Vadim Roytershteyn, Michael J. Anderson, Yushu Yao, and Prabhat. 2015. BD-CATS: big data clustering at trillion particle scale. In Proceedings of the International Conference for High Performance Computing, Networking, Storage and Analysis on - SC '15. ACM Press, New York, New York, USA, 1-12. https://doi.org/10.1145/2807591.2807616

[19] K. Sato, K. Mohror, A. Moody, T. Gamblin, B. R. d. Supinski, N. Maruyama, and S Matsuoka. 2014. A User-Level InfiniBand-Based File System and Checkpoint Strategy for Burst Buffers. In Cluster, Cloud and Grid Computing (CCGrid), 2014 14th IEEE/ACM International Symposium on. 21-30. https://doi.org/10.1109/CCGrid. 2014.24

[20] J.S. Vetter, S. Lee, D. Li, G. Marin, C. McCurdy, J. Meredith, P.C. Roth, and K. Spafford. 2014. Quantifying Architectural Requirements of Contemporary ExtremeScale Scientific Applications. In High Performance Computing Systems. Performance Modeling, Benchmarking and Simulation, S.A. Jarvis, S.A. Wright, and S.D. Hammond (Eds.). Lecture Notes in Computer Science, Vol. 8551. Springer International Publishing, 3-24. https://doi.org/10.1007/978-3-319-10214-6_1 